\title{
Salts of guanine and alkali metals: preparation and structural study at broad range of T, P
}

\author{
A.A. Gaydamaka ${ }^{1,2^{\star}}$, S.G. Arkhipov ${ }^{1,2}$, B.A. Zakharov ${ }^{1,2}$, Yu.V. Seryotkin ${ }^{1,3}$, E.V. Boldyreva ${ }^{1,2}$ \\ ${ }^{1}$ Novosibirsk State University, 630090, Novosibirsk, Russia, \\ ${ }^{2}$ Boreskov Institute of Catalysis SB RAS, 630090, Novosibirsk, Russia, \\ ${ }^{3}$ V.S. Sobolev Institute of Geology and Mineralogy SB RAS, 630090, Novosibirsk, Russia
}

a.gaidamaka@g.nsu.ru

High pressure is a powerful tool to study experimentally the response of selected hydrogen bonds to mechanical stress. Cooling is an alternative method to compress a structure. A comparison of compression on cooling and increasing pressure gives an insight into intermolecular interactions. Guanine and its derivatives, as well as nucleic acids, in general, attract much attention because of their interesting properties. Crystals made of small RNA or DNA fragments can serve as models of the effect of pressure on nucleic acids and oligonucleotides, similar to how the crystals of amino acids are used to model proteins. Nucleobases are the structural elements of nucleic acids. They are widely used as components of some crystalline drugs and molecular materials. Guanine is remarkable for its unique ability to form assemblies. In particular, oligonucleotides enriched with guanine can form quadruplexes in the presence of alkali and earth-alkaline metals. Because of the extremely low solubility of guanine in water and most of the organic solvents at neutral $\mathrm{pH}$, only a few guanine compounds are known. An additional challenge is to obtain single crystals. Crystal structures containing guanine, metal ions and water molecules can also be used, to shed more light on the interactions between the guanine anions, metal cations and water molecules. Potassium cations are of special biological importance because they form natural quadruplexes, which are present in telomeric parts of the chromosome. The hydrates of guanine metal salts are of interest in this respect. In this contribution, the approaches to the crystallization of salts of guanine and alkaline metals from aqueous, alcoholic and aqueous-alcoholic solutions. Two salts of guanine were investigated by single-crystal $\mathrm{X}$-ray diffraction, namely, $2 \mathrm{Na}^{+} \cdot \mathrm{C}_{5} \mathrm{H}_{3} \mathrm{~N}_{5} \mathrm{O}^{2-} \cdot 7 \mathrm{H}_{2} \mathrm{O}$ and $\mathrm{K}^{+} \cdot \mathrm{C}_{5} \mathrm{H}_{4} \mathrm{~N}_{5} \mathrm{O}^{-} \cdot \mathrm{H}_{2} \mathrm{O}$. The crystals of $\mathrm{K}^{+} \cdot \mathrm{C}_{5} \mathrm{H}_{4} \mathrm{~N}_{5} \mathrm{O}^{-} \cdot \mathrm{H} 2 \mathrm{O}$ were obtained for the first time. The structure is quite different from that of the previously documented sodium salt hydrate $\left(2 \mathrm{Na}^{+} \cdot \mathrm{C}_{5} \mathrm{H}_{3} \mathrm{~N}_{5} \mathrm{O}^{2-} \cdot 7 \mathrm{H}_{2} \mathrm{O}\right)$ [1]. The crystal structures of both sodium and potassium salt hydrates have channels. However, the structure of the channels, the cation coordination, the tautomeric form of the guanine anions, as well as the role of water molecules in the crystal structure are different for the two salt hydrates. In the potassium salt hydrate, there are two tautomeric forms of guanine anions and two types of potassium ions with different coordination. It is interesting to note, that though no "true" guanine quadruplexes could be found in the crystal structure of the potassium salt of guanine hydrate the "quartets" of guanine connected via hydrogen bonds with each other and two water molecules are present in this crystal structure. The sodium salt hydrate $\left(2 \mathrm{Na}^{+} \cdot \mathrm{C}_{5} \mathrm{H}_{3} \mathrm{~N}_{5} \mathrm{O}^{2-} \cdot 7 \mathrm{H}_{2} \mathrm{O}\right)$ was characterized by single-crystal X-ray diffraction in the pressure range of $1 \mathrm{~atm}-2.5 \mathrm{GPa}$ [2] as well as in the temperature range $100 \mathrm{~K}-300 \mathrm{~K}$. The potassium salt of guanine was characterized by single-crystal X-ray diffraction in the temperature range $100 \mathrm{~K}-300 \mathrm{~K}$. ThetaToTensor software was used to calculate the coefficients of thermal expansion tensor and create a graphical representation of the characteristic surface [3]. The anisotropy of strain on temperature variation was compared for the two salt hydrates, the similarities and the differences are discussed concerning the intermolecular interactions [4].

[1] Gur D., Shimon L. J. W. (2015) Acta Crystallographica Section E: Crystallographic Communications, 71 (3), $281-283$.

[2] A.Gaydamaka et al. (2019) CrystEngComm , 21, 4484-92.

[3] Bubnova, R. S., V. A. Firsova, and S. K. Filatov. (2013) Glass Physics and Chemistry, 39.3, 347-350.

[4] Gaydamaka, A. A., Arkhipov, S. G., Boldyreva, E. V., 2021, Acta Crystallographica Section B, in preparation.

Keywords: guanine, nucleobase, single crystal X-ray diffraction, XRD, vibrational spectroscopy, high pressure, low temperature, ionic channels. 